\title{
ON THE GROWTH OF THE INTEGRAL MEANS OF SUBHARMONIC FUNCTIONS OF ORDER LESS THAN ONE ${ }^{1}$
}

BY

FARUK F. ABI-KHUZAM

ABSTRACT. Let $u$ be a subharmonic function of order $\lambda(0<\lambda<1)$, and let $m_{s}(r, u)=\left\{(1 / 2 \pi) \int_{-\pi}^{\pi}\left|u\left(r e^{i \theta}\right)\right|^{s} d \theta\right\}^{1 / s}$. We compare the growth of $m_{s}(r, u)$ with that of the Riesz mass of $u$ as measured by $N(r, u)=$ $(1 / 2 \pi) \int_{-\pi}^{\pi} u\left(r e^{i \theta}\right) d \theta$. A typical result of this paper states that the following inequality is sharp:

$$
\liminf _{r \rightarrow \infty} \frac{m_{s}(r, u)}{N(r, u)}<m_{s}\left(\psi_{\lambda}\right)
$$

where $\psi_{\lambda}(\theta)=(\pi \lambda / \sin \pi \lambda) \cos \lambda \theta$.

The case $s=1$ is due to Edrei and Fuchs, the case $s=2$ is due to Miles and Shea and the case $s=\infty$ is due to Valiron.

Introduction. Let $f$ be a meromorphic function of finite order $\lambda$ and let $\log M(r, f), T(r, f), N(r, 0), N(r, \infty)$ be the basic functionals in Nevanlinna theory associated with $f$. The problems of finding sharp asymptotic inequalities for ratios of these functionals originated and were investigated by Valiron [14], Polya, Nevanlinna and others.

Recently, Miles and Shea [10, p. 377] used Fourier series techniques to obtain sharp bounds for an $L_{2}$ version of these problems. They used their result to get the best bounds yet in the $L_{1}$ case-very close to the conjectured sharp bound for this still open problem due to Nevanlinna [11, p. 54]. When the order of $f$ is less than one the Nevanlinna problem was completely solved by Edrei and Fuchs [5] and for entire functions of any finite order with zeroes on a ray by Hellerstein and Williamson [8].

In this paper we consider an $L_{s}(1 \leqslant s<\infty)$ version of these problems:

Let $u$ be a subharmonic function in the plane. Put

$$
\begin{aligned}
& m_{s}(r, u)=\left\{\frac{1}{2 \pi} \int_{-\pi}^{\pi}\left|u\left(r e^{i \theta}\right)\right|^{s} d \theta\right\}^{1 / s}, \\
& N(r, u)=\frac{1}{2 \pi} \int_{-\pi}^{\pi} u\left(r e^{i \theta}\right) d \theta
\end{aligned}
$$

Received by the editors July 31, 1975 and, in revised form, February 4, 1977.

AMS (MOS) subject classifications (1970). Primary 30A64, 31A10; Secondary 30A68.

Key words and phrases. Riesz mass, order of a subharmonic function, proximate order.

1 This research was supported in part by NSF Grant A78-8319.

(1) American Mathematical Society 1978 
and consider the following

Problem 1. Determine sharp upper bounds for

$$
\liminf _{r \rightarrow \infty} \frac{m_{s}(r, u)}{N(r, u)} \quad(1 \leqslant s<\infty)
$$

where $u$ is a subharmonic function of finite nonintegral order $\lambda$.

Problem 2. Determine sharp lower bounds for

$$
\limsup _{r \rightarrow \infty} \frac{m_{s}(r, u)}{N(r, u)} \quad(1 \leqslant s<\infty)
$$

where $u$ is a subharmonic function whose Riesz mass is distributed along a ray and whose order is finite.

By combining the methods of Edrei and Fuchs and Miles and Shea, I have obtained a complete solution of Problem 1 for subharmonic functions of order less than one, and for a class of $\Delta$-subharmonic functions of order less than one. I have also obtained a complete solution of Problem 2. For functions of order greater than one, Problem 1 remains unsolved.

In concluding this introduction, I wish to express my sincere gratitude to Professor Albert Edrei; most of the ideas in this paper were developed while I was a student under his guidance. I am also grateful to the referee for various suggestions and comments to improve this paper.

1. Summary and notation. Consider a function $w=u-v$, where $u$ and $v$ are subharmonic in the plane and harmonic in a neighbourhood of the origin. Let $\mu$ and $\nu$ be the Riesz masses of $u$ and $v$ respectively, and let

$$
n(t, u)=\int_{|a|<t} d \mu(a), \quad n(t, v)=\int_{|a|<t} d v(a) .
$$

Define $N(r, \cdot)$ by

$$
N(r, \cdot)=\int_{0}^{r} n(t, \cdot) t^{-1} d t
$$

and put

$$
T(r)=T(r, w)=\frac{1}{2 \pi} \int_{-\pi}^{\pi} w^{+}\left(r e^{i \theta}\right) d \theta+N(r, v) .
$$

$T(r)$ is called the characteristic of $w$; the order $\lambda$ and the lower order $\mu$ of $w$ are defined in terms of $T(r)$ by:

$$
\lambda=\limsup _{r \rightarrow \infty} \frac{\log T(r)}{\log r}, \quad \mu=\liminf _{r \rightarrow \infty} \frac{\log T(r)}{\log r} .
$$

(This double use of the letter $\mu$ should not give rise to any confusion.)

Although $T(r)$ as defined by (1.3) is not unique, it is an easy matter to obtain a unique characteristic for the function $w$. Indeed, all that is needed is 
to be able to construct subharmonic functions $u$ and $v$ such that their Riesz masses are respectively equal to the positive and negative parts of the Riesz mass of $w$ and then define the characteristic from this special representation $w=u-v$.

From now on we shall assume that this has been done. Thus under consideration is a ( $\delta$-subharmonic) function $w=u-v$ where,

(i) $u$ and $v$ are subharmonic in the plane and harmonic in a neighbourhood of the origin with $w(0)=0$;

(ii) the Riesz mass $\mu$ of $u$ equals the positive part of the Riesz mass of $w$, and the Riesz mass $\nu$ of $v$ equals the negative part of the Riesz mass of $w$;

(iii) the order $\lambda$ of $w$ is finite and nonintegral.

We denote by $\mathscr{D}$ the class of all functions $w$ satisfying (i), (ii) and (iii).

For $w \in \mathscr{D}$, define $\alpha_{m}$ by

$$
w(z)=\operatorname{Re}\left\{\sum_{m=1}^{\infty} \alpha_{m} z^{m}\right\}
$$

for $z$ near 0 .

Since the order $\lambda$ of $w$ is finite, we may then write

$$
\begin{aligned}
w(z)= & \operatorname{Re}(p(z))+\int_{|a|<\infty} \log |E(z / a, q)| d \mu(a) \\
& -\int_{|a|<\infty} \log |E(z / a, q)| d \nu(a) ;
\end{aligned}
$$

where $q=[\lambda], p(z)=\alpha_{q} z^{q}+\cdots+\alpha_{1} z$, and $E(x, q)$ is the Weierstrass primary factor of genus $q$.

The characteristic of $w$ as defined in (1.3) was introduced by Privaloff [12] who also established the following:

(a) $T(r, w)$ is a nondecreasing function of $r$;

(b) $T(r, w)=o(\log r)$ implies that $w$ is a constant;

(c) $T(r, w)=O(\log r)$ is a necessary and sufficient condition for $w$ to have the form

$$
w(z)=\int \log |z-a| d \mu(a)-\int \log |z-a| d \nu(a)+\text { constant }
$$

where the mass distributions $\mu$ and $\nu$ are bounded.

The properties (a), (b) and (c) show that $T(r)$ gives a great deal of information about the function $w$ and lead naturally to the consideration of functions $w$ of finite order defined by (1.4).

In order to state our results we use the notation $\psi_{\lambda}(\theta)=\pi \lambda \csc \pi \lambda \cos (\lambda \theta)$; then we have

THEOREM 1. Let $w \in \mathscr{D}$ be subharmonic of order $\lambda(0<\lambda<1)$; then 
This inequality is sharp.

$$
\liminf _{r \rightarrow \infty} \frac{m_{s}(r, u)}{N(r, u)} \leqslant m_{s}\left(\psi_{\lambda}\right) \quad(1 \leqslant s<\infty) .
$$

THEOREM 2. Let $w \in \mathscr{D}$ be subharmonic. If the Riesz mass of $w$ is distributed along $a$ ray and if its order $\lambda$ is nonintegral, then

$$
\limsup _{r \rightarrow \infty} \frac{m_{s}(r, u)}{N(r, u)} \geqslant m_{s}\left(\psi_{\lambda}\right) \quad(1 \leqslant s<\infty) .
$$

This inequality is sharp.

Theorems 1 and 2 both hold true when $w$ and $\psi_{\lambda}$ are replaced by $w^{+}$and $\psi_{\lambda}^{+}$respectively. Also, both theorems hold true for $w \in \mathscr{D},(0<\lambda<1)$ satisfying the condition $N(r, u)=N(r, v)$; the result corresponding to Theorem 2 requiring the additional assumption that the masses $\mu$ and $\nu$ be distributed along the negative and positive $x$-axes respectively. Since the condition $N(r, u)=N(r, v)$ is somewhat artificial we omit the proofs.

For a general $\Delta$-subharmonic function the following result will follow easily from theorems of Hardy and Littlewood [7]:

Theorem 3. Let $w \in \mathcal{D}$ be of nonintegral order $\lambda$. Denote by $\left\{c_{m}(r)\right\}$ the Fourier coefficients of $w$ and put $\tilde{w}(z)=w(z)-\Sigma_{|m| \leqslant q} c_{m}(r) e^{i m \theta}$. Then

$$
\liminf _{r \rightarrow \infty} \frac{m_{s}(r, \tilde{w})}{N(r)} \leqslant m_{s}\left(\tilde{\psi}_{\lambda}\right)
$$

where $N(r)=N(r, u)+N(r, v), q=[\lambda], s=2 k, k=1,2,3, \ldots$ and $\tilde{\psi}_{\lambda}$ is defined analogously to $\tilde{w}$. Furthermore (1.9) is sharp.

We point out that the proof of Theorem 2 carries over to higher dimensions provided that appropriate restrictions are put on the index $s$. For example, if $u$ is subharmonic in $R^{m}$ where $m=3$ or 4 , then an analogue of Theorem 2 may be obtained for the range $1 \leqslant s \leqslant 2$.

2. Preliminary lemmas. Let $w \in \mathcal{D}$ and denote by $c_{m}(r)=c_{m}(r, w)$ the Fourier coefficients of $w$, i.e.,

$$
c_{m}(r)=\frac{1}{2 \pi} \int_{-\pi}^{\pi} w\left(r e^{i \theta}\right) e^{-i m \theta} d \theta .
$$

Using (1.6) it is possible to compute these coefficients [10], and one finds that

$$
\begin{gathered}
c_{m}(r)=\frac{1}{2} \alpha_{m} r^{m}+\frac{1}{2 m} \int_{|a|<r}\left\{\left(\frac{r}{a}\right)^{m}-\left(\frac{\bar{a}}{r}\right)^{m}\right\} d \mu(a) \\
-\frac{1}{2 m} \int_{|a|<r}\left\{\left(\frac{r}{a}\right)^{m}-\left(\frac{\bar{a}}{r}\right)^{m}\right\} d \nu(a)
\end{gathered}
$$


for $m \geqslant 1$ and, for $m \geqslant q+1$, also

$$
\begin{aligned}
c_{m}(r)=\frac{1}{2 m} & \left\{\int_{|a|>r}\left(\frac{r}{a}\right)^{m} d \mu(a)-\int_{|a|>r}\left(\frac{r}{a}\right)^{m} d \nu(a)\right. \\
& \left.+\int_{|a|<r}\left(\frac{\bar{a}}{r}\right)^{m} d \mu(a)-\int_{|a|<r}\left(\frac{\bar{a}}{r}\right)^{m} d \nu(a)\right\},
\end{aligned}
$$

where $q=[\lambda]$ and $\lambda$ is the order of $w$.

For $m<0, c_{m}(r)=\overline{c_{-m}(r)}$, and $c_{0}(r)=N(r, u)-N(r, v)$.

From (2.2) and (2.3) one easily obtains [10]

$$
\begin{aligned}
& \left|c_{0}(r)\right| \leqslant N(r), \\
& \left|c_{m}(r)\right| \leqslant \frac{1}{2}\left|\alpha_{m}\right| r^{m}+\frac{1}{2} m \int_{0}^{r}\left\{(r / t)^{m}-(t / r)^{m}\right\} N(t) d t / t+N(r)
\end{aligned}
$$

$1 \leqslant m \leqslant q, q \neq 0$, and when $m \geqslant q+1$

$$
\left|c_{m}(r)\right| \leqslant \frac{1}{2} m\left\{\int_{0}^{r}(t / r)^{m} N(t) d t / t+\int_{r}^{\infty}(r / t)^{m} N(t) d t / t\right\}-N(r),
$$

where $N(r)=N(r, u)+N(r, v)$.

LeMma 2.1. Let $c_{m}(r)$ be the Fourier coefficients of a function $w \in \mathscr{D}$ of nonintegral order $\lambda$. Then there exists a slowly varying function $L$, a sequence $r_{n}$ increasing to infinity, and two absolute constants $M$ and $r_{0}$, such that

$$
\begin{array}{ll}
N(t) \leqslant t^{\lambda} L(t)=A(t) & (0<t<\infty) ; \\
N\left(r_{n}\right)=A\left(r_{n}\right) ; & \\
\frac{\left|c_{m}(r)\right|}{A(r)} \leqslant \frac{M}{|m|+1} \quad\left(r \geqslant r_{0}\right) .
\end{array}
$$

Here slowly varying means that $L$ is positive and satisfies

$$
\lim _{r \rightarrow \infty} L(\sigma r) / L(r)=1
$$

for every $\sigma>0$.

LEMMA 2.2 [6]. Let there be given two functions $\varphi_{1}(x)$ and $\varphi_{2}(x)$ defined on the interval $0 \leqslant x<\infty$, with $\varphi_{2}(x) \geqslant 0$. Let there also be given two numbers $\lambda \geqslant 0$ and $\varepsilon>0$ such that

(a) both of the integrals

$$
\int_{0}^{\infty} \frac{\left|\varphi_{1}(x)\right|}{x^{\sigma+1}} d x \text { and } \int_{0}^{\infty} \frac{\varphi_{2}(x)}{x^{\sigma+1}} d x
$$

converge for $\lambda<\sigma<\lambda+\varepsilon$, and the second integral diverges for $\sigma<\lambda$;

(b) there exists a function $\Psi(z)$, holomorphic in $|z-\lambda|<\varepsilon$ and real for real $z$, such that for $\lambda<\sigma<\lambda+\varepsilon$ 


$$
\int_{0}^{\infty} \frac{\varphi_{1}(r)}{r^{\sigma+1}} d r=\Psi(\sigma) \int_{0}^{\infty} \frac{\varphi_{2}(r)}{r^{\sigma+1}} d r
$$

Then

$$
\limsup _{r \rightarrow \infty} \frac{\varphi_{1}(r)}{\varphi_{2}(r)} \geqslant \Psi(\lambda) \geqslant \liminf _{r \rightarrow \infty} \frac{\varphi_{1}(r)}{\varphi_{2}(r)} .
$$

Definition [2, p. 149]. Let $g$ be a real valued integrable function on $[-\pi, \pi]$. The "star function" of $g$ is defined by

$$
g^{*}(\theta)=\sup _{|E|=2 \theta} \int_{E} g \quad(0<\theta<\pi,|E|=\text { Lebesgue measure of } E) .
$$

LEMma $2.3\left[2\right.$, p. 150]. For $g, h \in L_{\mathrm{l}}[-\pi, \pi]$ the following statements are equivalent.

(a) For every convex nondecreasing function $\Phi$ on $(-\infty, \infty)$

$$
\int_{-\pi}^{\pi} \Phi(g(x)) d x \leqslant \int_{-\pi}^{\pi} \Phi(h(x)) d x
$$

$$
g^{*}(\theta)<h^{*}(\theta) \quad(0<\theta<\pi) .
$$

Proof of Lema 2.1. The existence of the function $A(t)$ and the sequence $r_{n}$ is Theorem 16 of $[9$, p. 35]. The proof of (c) is given in [1]. We add here that, since $w$ is harmonic in a neighborhood of the origin (property (i) of the class $\mathscr{D}), N(t)=N(t, u)+N(t, v)$ vanishes in a neighborhood of the origin, and so, we may and do take $A(t)$ to be vanishing in the same neighborhood of the origin.

3. Proof of Theorem 1. Let $w \in \mathcal{D}$ be subharmonic of order $\lambda(0<\lambda<1)$, and let $N(r)=N(r, w)$. Then the order of $N(r)$ is also equal to $\lambda$. Let $\left\{r_{n}\right\}$ be the sequence increasing to infinity and satisfying parts (b) and (c) of Lemma 2.1. By part (c), there is a subsequence (which we also denote by $\left\{r_{n}\right\}$ ) and numbers $\xi_{m}$ such that

$$
\frac{c_{m}\left(r_{n}\right)}{A\left(r_{n}\right)} \rightarrow \xi_{m} \quad\left(r_{n} \rightarrow \infty, \text { all } m\right) .
$$

Clearly then $\xi_{m}=O\left(m^{-1}\right)$, and so, by the Riesz-Fisher theorem, there is a function $\varphi \in L_{2}(-\pi, \pi)$ such that

$$
\varphi(\theta) \sim \sum_{m=-\infty}^{\infty} \xi_{m} e^{i m \theta} .
$$

If $1<s<2$, then using the fact that the $L_{s}(-\pi, \pi)$ norm is a nondecreasing function of $s$, together with Parseval's identity, (3.1) and part (c) of Lemma 2.1, we have 


$$
\begin{aligned}
\underset{r_{n} \rightarrow \infty}{\lim \sup }\left\{\frac{1}{2 \pi} \int_{-\pi}^{\pi}\left|\frac{w\left(r_{n} e^{i \theta}\right)}{A\left(r_{n}\right)}-\varphi(\theta)\right|^{s} d \theta\right\}^{1 / s} \\
\quad \leqslant \limsup _{r_{n} \rightarrow \infty}\left\{\sum_{m=-\infty}^{\infty}\left|\frac{c_{m}\left(r_{n}\right)}{A\left(r_{n}\right)}-\xi_{m}\right|^{2}\right\}^{1 / 2}=0 .
\end{aligned}
$$

If $2 \leqslant s<\infty$, then applying the Hausdorff-Young theorem, (3.1), and taking into account part (c) of Lemma 2.1 we obtain:

$$
\begin{aligned}
\underset{r_{n} \rightarrow \infty}{\lim \sup _{0}}\left\{\frac{1}{2 \pi} \int_{-\pi}^{\pi}\left|\frac{w\left(r_{n} e^{i \theta}\right)}{A\left(r_{n}\right)}-\varphi(\theta)\right|^{s} d \theta\right\}^{1 / s} \\
\quad \leqslant \limsup _{r_{n} \rightarrow \infty}\left\{\sum_{m=-\infty}^{\infty}\left|\frac{c_{m}\left(r_{n}\right)}{A\left(r_{n}\right)}-\xi_{m}\right|^{s^{\prime}}\right\}^{1 / s^{\prime}}=0
\end{aligned}
$$

where $1 / s+1 / s^{\prime}=1$.

From (3.3), (3.4), Minkowski's inequality and part (b) of Lemma 2.1, we conclude:

$$
\begin{aligned}
& \lim _{r_{n} \rightarrow \infty} \frac{m_{s}\left(r_{n}, w\right)}{N\left(r_{n}\right)}=m_{s}(|\varphi|) \quad(1 \leqslant s<\infty) ; \\
& \lim _{r_{n} \rightarrow \infty} \frac{m_{s}\left(r_{n}, w^{+}\right)}{N\left(r_{n}\right)}=m_{s}\left(\varphi^{+}\right) \quad(1 \leqslant s<\infty) ;
\end{aligned}
$$

and

$$
\lim _{r_{n} \rightarrow \infty}\left\{\frac{1}{2 \pi} \int_{E}\left|\frac{w\left(r_{n} e^{i \theta}\right)}{A\left(r_{n}\right)}-\varphi(\theta)\right|^{s} d \theta\right\}^{1 / s}=0
$$

for any measurable set $E(\subset[-\pi, \pi])$ and any $s(1 \leqslant s<\infty)$.

It follows from (3.5) and (3.6) that Theorem 1 will be established if we show that

$$
m_{s}\left(\varphi^{+}\right)<m_{s}\left(\psi_{\lambda}^{+}\right)
$$

and

$$
m_{s}(|\varphi|)<m_{s}\left(\left|\psi_{\lambda}\right|\right) .
$$

Proof of (3.8). Let $E \subset[-\pi, \pi]$ be a Lebesgue measurable set of measure $2 \beta(0<\beta<\pi)$. From (3.7) we have 


$$
\frac{1}{2 \pi} \int_{E} \varphi(\theta) d \theta=\lim _{r_{n} \rightarrow \infty} \frac{1}{A\left(r_{n}\right)} \cdot \frac{1}{2 \pi} \int_{E} w\left(r_{n} e^{i \theta}\right) d \theta .
$$

To estimate the right-hand side of (3.10) we follow the well-established methods of Edrei and Fuchs [5]:

Since the function $\log \left|1+r e^{i \theta} /\right| a||$ is an even function of $\theta$ which decreases steadily as $\theta$ varies from 0 to $\pi$, we have [3, p. 15],

$$
\frac{1}{2 \pi} \int_{E} \log \left|1-\frac{r e^{i \theta}}{a}\right| d \theta \leqslant \frac{1}{\pi} \int_{0}^{\beta} \log \left|1+\frac{r e^{i \theta}}{|a|}\right| d \theta .
$$

Using this in (1.6) with $q=0$ and $d \nu(a) \equiv 0$, we deduce

$$
\begin{aligned}
\frac{1}{2 \pi} \int_{E} w\left(r e^{i \theta}\right) d \theta & \leqslant \int_{|a|<\infty} d \mu(a) \frac{1}{\pi} \int_{0}^{\beta} \log \left|1+\frac{r e^{i \theta}}{|a|}\right| d \theta \\
& =\int_{0}^{\infty} N(t) P(t, r, \beta) d t
\end{aligned}
$$

where $P(t, r, \beta)=r \sin \beta /\left(t^{2}+2 t r \cos \beta+r^{2}\right), 0<\beta<\pi$.

Using parts (a) and (b) of Lemma 2.1 and properties of proximate orders we deduce from (3.10) and (3.11), that

$$
\begin{aligned}
\frac{1}{2 \pi} \int_{E} \varphi(\theta) d \theta & \leqslant \lim _{r_{n} \rightarrow \infty} \frac{1}{A\left(r_{n}\right)} \int_{0}^{\infty} A(t) P\left(t, r_{n}, \beta\right) d t \\
& =\lim _{r \rightarrow \infty} \frac{1}{A(r)} \int_{0}^{\infty} A(t) P(t, r, \beta) d t \\
& =\frac{\sin \lambda \beta}{\sin \pi \lambda}=\frac{1}{2 \pi} \int_{-\beta}^{\beta} \psi_{\lambda}(\theta) d \theta
\end{aligned}
$$

In view of the definition of the star function and the fact that $\psi_{\lambda}$ decreases steadily from 0 to $\pi,(3.12)$ implies

$$
\varphi^{*}(\beta) \leqslant \psi_{\lambda}^{*}(\beta), \quad 0<\beta<\pi .
$$

It is easily seen that (3.13) remains true for $\beta=0$ and $\beta=\pi$; thus, applying Lemma 2.2 with $g=\varphi, h=\psi_{\lambda}$ and $\Phi(x)=(\max (x, 0))^{s}$ we see that $(3.8)$ is an immediate consequence of (3.13).

Proof of (3.9). The proof of (3.9) will be along the same lines as the proof of (3.8) but a little extra care is needed.

Let $E \subset[-\pi, \pi]$ be a Lebesgue measurable set of measure $2 \beta(0<\beta<$ $\pi)$. Put $E_{1}=E_{1}(r)=\left\{\theta \in E: w\left(r e^{i \theta}\right) \geqslant 0\right\}, E_{2}=E_{2}(r)=\left\{\theta \in E: w\left(r e^{i \theta}\right)\right.$ $<0\}$, and $2 \beta_{1}(r)=\left|E_{1}\right|, 2 \beta_{2}(r)=\left|E_{2}\right|$. Thus $\beta_{1}(r)+\beta_{2}(r)=\beta$ for all $r$.

Again from (3.7) we have

$$
\frac{1}{2 \pi} \int_{E}|\varphi(\theta)| d \theta=\lim _{r_{n} \rightarrow \infty} \frac{1}{A\left(r_{n}\right)} \cdot \frac{1}{2 \pi} \int_{E}\left|w\left(r_{n} e^{i \theta}\right)\right| d \theta .
$$


Now we write $\int_{E}\left|w\left(r e^{i \theta}\right)\right| d \theta=\int_{E_{1}} w\left(r e^{i \theta}\right) d \theta-\int_{E_{2}} w\left(r e^{i \theta}\right) d \theta$ and follow the same steps that led to (3.11). At one point we need to use the fact that

$$
\begin{aligned}
\frac{1}{2 \pi} \int_{E_{2}}-\log \left|1-\frac{r e^{i \theta}}{a}\right| d \theta & \leqslant \frac{1}{\pi} \int_{0}^{\beta_{2}}-\log \left|1-\frac{r e^{i \theta}}{|a|}\right| d \theta \\
& =-\log ^{+}(r /|a|)+\frac{1}{\pi} \int_{0}^{\pi-\beta_{2}} \log \left|1+\frac{r e^{i \theta}}{|a|}\right| d \theta
\end{aligned}
$$

and we are led to

$$
\begin{aligned}
\frac{1}{2 \pi} \int_{E}\left|w\left(r e^{i \theta}\right)\right| d \theta \leqslant & \int_{0}^{\infty} N(t) P\left(t, r, \beta_{1}(r)\right) d t-N(r) \\
& +\int_{0}^{\infty} N(t) P\left(t, r, \pi-\beta_{2}(r)\right) d t .
\end{aligned}
$$

It is our intention to set $r=r_{n}$, but before doing so we select (if necessary) subsequences and assume that $\beta_{1}\left(r_{n}\right) \rightarrow \beta_{1}, \beta_{2}\left(r_{n}\right) \rightarrow \beta_{2}$. We shall also assume that $\beta_{1}>0, \beta_{2}>0$, so that for large $n, 0<\beta_{1}\left(r_{n}\right)<\pi, 0<\beta_{2}\left(r_{n}\right)<\pi$ and of course, $\beta_{1}\left(r_{n}\right)+\beta_{2}\left(r_{n}\right)=\beta$. Now in (3.15) putting $r=r_{n}$ and taking account of (3.14) and properties of proximate orders we are led to the following inequality:

$$
\begin{aligned}
& \frac{1}{2 \pi} \int_{E}|\varphi(\theta)| d \theta \\
& \leqslant \lim _{r_{n} \rightarrow \infty} \frac{1}{A\left(r_{n}\right)}\left\{\int _ { 0 } ^ { \infty } A ( t ) \left(P\left(t, r_{n}, \beta_{1}\left(r_{n}\right)\right)\right.\right. \\
& \left.\left.\quad+P\left(t, r_{n}, \pi-\beta_{2}\left(r_{n}\right)\right)\right) d t-A\left(r_{n}\right)\right\} \\
& =\frac{\sin \lambda \beta_{1}}{\sin \pi \lambda}+\frac{\sin \lambda\left(\pi-\beta_{2}\right)}{\sin \pi \lambda}-1 \\
& =\frac{1}{\pi} \int_{0}^{\beta_{1}} \psi_{\lambda}(\theta) d \theta+\frac{1}{\pi} \int_{0}^{\pi-\beta_{2}} \psi_{\lambda}(\theta) d \theta-1 \\
& =\frac{1}{\pi} \int_{0}^{\beta_{1}} \psi_{\lambda}(\theta) d \theta-\frac{1}{\pi} \int_{\pi-\beta_{2}}^{\pi} \psi_{\lambda}(\theta) d \theta \\
& \leqslant \frac{1}{\pi} \int_{0}^{\beta_{1}}\left|\psi_{\lambda}(\theta)\right| d \theta+\frac{1}{\pi} \int_{\pi-\beta_{2}}^{\pi}\left|\psi_{\lambda}(\theta)\right| d \theta \\
& \leqslant \frac{1}{2 \pi}\left|\psi_{\lambda}\right|^{*}\left(\beta_{1}+\beta_{2}\right)=\frac{1}{2 \pi}\left|\psi_{\lambda}\right|^{*}(\beta) .
\end{aligned}
$$

From (3.16) follows that

$$
|\varphi|^{*}(\beta) \leqslant\left|\psi_{\lambda}\right|^{*}(\beta), \quad 0<\beta<\pi .
$$


The inequality (3.17) was established under the assumption $\beta_{1} \beta_{2} \neq 0$. If $\beta_{2}=0$, then it is possible to have $\beta_{2}\left(r_{n}\right)=0$ for infinitely many values of $n$. In this case $\beta_{1}\left(r_{n}\right)=\beta,(1 / 2 \pi) \int_{E}\left|w\left(r_{n} e^{i \theta}\right)\right| d \theta=(1 / 2 \pi) \int_{E_{1}} w\left(r_{n} e^{i \theta}\right) d \theta$ and then (3.17) follows from (3.13).

If $\beta_{1}=0$ and $\beta_{1}\left(r_{n}\right)=0$ for infinitely many values of $n$, then

$$
\begin{aligned}
\frac{1}{2 \pi} \int_{E}\left|w\left(r_{n} e^{i \theta}\right)\right| d \theta & =\frac{1}{2 \pi} \int_{E_{2}}-w\left(r_{n} e^{i \theta}\right) d \theta \\
& =-A\left(r_{n}\right)+\frac{1}{2 \pi} \int_{E \xi} w\left(r_{n} e^{i \theta}\right) d \theta \\
& <-A\left(r_{n}\right)+\int_{0}^{\infty} N(t) P\left(t, r_{n}, \pi-\beta\right) d t .
\end{aligned}
$$

This gives

$$
\frac{1}{2 \pi} \int_{E}|\varphi(\theta)| d \theta \leqslant-1+\frac{1}{2 \pi} \int_{0}^{\pi-\beta} \psi_{\lambda}(\theta) d \theta=-\frac{1}{\pi} \int_{\pi-\beta}^{\pi} \psi_{\lambda}(\theta) d \theta
$$

which is only possible if $\frac{1}{2}<\lambda<1$ in which case it again leads to $|\varphi|^{*}(\beta) \leqslant$ $\left|\psi_{\lambda}\right|^{*}(\beta)$.

The validity of (3.17) for $\beta=0$ is trivial; also, since for all $g \geqslant 0, g$ is increasing and convex, it follows from inequality (3.17) that $|\varphi|^{*}(\pi) \leqslant$ $\left|\psi_{\lambda}\right|^{*}(\pi)$

Applying Lemma 2.3 with $g=|\varphi|, h=\left|\psi_{\lambda}\right|$ and $\Phi(x)=(\max (x, 0))^{s}$, (3.9) follows immediately from (3.17).

4. Proof of Theorem 2. Let $w \in \mathscr{D}$ be subharmonic of nonintegral order $\lambda$ and assume that the Riesz mass of $w$ is distributed along the negative real axis. In this case the Fourier coefficients $\gamma_{m}(r)$ of $w$ are given by:

$$
\begin{aligned}
(-1)^{m} \gamma_{m}(r)= & \frac{(-1)^{m}}{2} \alpha_{m} r^{m} \\
& +\frac{1}{2} m \int_{0}^{r}\left\{\left(\frac{r}{t}\right)^{m}-\left(\frac{t}{r}\right)^{m}\right\} N(t) \frac{d t}{t}+N(r)
\end{aligned}
$$

where $1 \leqslant m \leqslant q, q \neq 0$ and $q=[\lambda]$; and

$$
\begin{array}{r}
(-1)^{m} \gamma_{m}(r)=N(r)-\frac{m}{2}\left\{\int_{0}^{r}\left(\frac{t}{r}\right)^{m} N(t) \frac{d t}{t}+\int_{r}^{\infty}\left(\frac{r}{t}\right)^{m} N(t) \frac{d t}{t}\right\} \\
(m \geqslant q+1) .
\end{array}
$$

A result of Edrei and Fuchs [4, p. 308] implies that functions $w$ satisfying the hypothesis of Theorem 2, also satisfy

$$
r^{q}=o(T(r, w)) \quad(r \rightarrow \infty) .
$$


From (4.3) follows easily that

$$
r^{q}=o\left(m_{s}(r, w)\right), \quad r^{q}=o\left(m_{s}\left(r, w^{+}\right)\right) \quad(1 \leqslant s<\infty, r \rightarrow \infty)
$$

and (4.4) together with some standard computations implies that we may assume, in the proof of Theorem 2 , that $\alpha_{m}=0$ for $1 \leqslant m \leqslant q$. From now on we make this assumption.

Let $\sigma$ be any real number satisfying $\lambda<\sigma<q+1$. Then a simple integration by parts of (4.1) and (4.2) gives:

$$
\int_{0}^{\infty} \frac{\gamma_{m}(r)}{r^{\sigma+1}} d r=\frac{(-1)^{m} \sigma^{2}}{\sigma^{2}-m^{2}} \int_{0}^{\infty} \frac{N(r)}{r^{\sigma+1}} d r
$$

from which follows

$$
\int_{0}^{\infty} \frac{w\left(r e^{i \theta}\right)}{r^{\sigma+1}} d r=\psi_{\sigma}(\theta) \int_{0}^{\infty} \frac{N(r)}{r^{\sigma+1}} d r
$$

where $N(r)=N(r, w)$ and $-\pi \leqslant \theta \leqslant \pi$.

Let $s$ be a real number satisfying $1<s<\infty$ (the case $s=1$ is well known) and let $s^{\prime}$ be the index conjugate to $s$, i.e., $1 / s^{\prime}+1 / s=1$. Let $g(\theta)$ be a real-valued function, continuous on $[-\pi, \pi]$ and such that

$$
m_{s^{\prime}}(g)=\left\{\frac{1}{2 \pi} \int_{-\pi}^{\pi}|g(\theta)|^{s^{\prime}} d \theta\right\}^{1 / s^{\prime}}=1 .
$$

From (4.6) we calculate

$$
\begin{aligned}
\int_{0}^{\infty} \frac{(1 / 2 \pi) \int_{-\pi}^{\pi} w\left(r e^{i \theta}\right) g(\theta) d \theta}{r^{\sigma+1}} d r \\
=\left(\frac{1}{2 \pi} \int_{-\pi}^{\pi} \psi_{\sigma}(\theta) g(\theta) d \theta\right) \int_{0}^{\infty} \frac{N(r)}{r^{\sigma+1}} d r \\
=\Psi(\sigma) \int_{0}^{\infty} \frac{N(r)}{r^{\sigma+1}} d r .
\end{aligned}
$$

The continuity of $g(\theta)$ ensures that $\Psi(\sigma)=\Psi(\sigma ; g)$ is holomorphic in a sufficiently small neighbourhood of $\lambda$. All the other conditions of Lemma 2.2 are satisfied and so

$$
\limsup _{r \rightarrow \infty} \frac{1}{N(r)} \cdot \frac{1}{2 \pi} \int_{-\pi}^{\pi} w\left(r e^{i \theta}\right) g(\theta) d \theta \geqslant \Psi(\lambda) .
$$

Minkowski's inequality, (4.7) and (4.9) give

$$
\limsup _{r \rightarrow \infty} \frac{m_{s}(r, w)}{N(r)} \geqslant \Psi(\lambda)=\Psi(\lambda ; g)
$$

valid for any continuous function $g(\theta)$ that satisfies (4.7). Since the 
continuous functions are dense in $L_{s^{\prime}}[-\pi, \pi]$ we conclude from (4.10) that

$$
\limsup _{r \rightarrow \infty} \frac{m_{s}(r, w)}{N(r)} \geqslant \sup _{m_{s}^{\prime}(g)=1}|\Psi(\lambda ; g)|=m_{s}\left(\psi_{\lambda}\right)
$$

and the proof of (1.8) is complete.

Now let $g(\theta)$ be a real-valued function, continuous and nonnegative on [- $\pi, \pi]$ and satisfying (4.7). Let $E_{\lambda}=\left\{\theta: \psi_{\lambda}(\theta) \geqslant 0\right\}$. From (4.6) we calculate

$$
\begin{aligned}
\int_{0}^{\infty} \frac{(1 / 2 \pi) \int_{E_{\lambda}} w\left(r e^{i \theta}\right) g(\theta) d \theta}{r^{\sigma+1}} d r \\
=\left(\frac{1}{2 \pi} \int_{E_{\lambda}} \psi_{\lambda}(\theta) g(\theta) d \theta\right) \int_{0}^{\infty} \frac{N(r)}{r^{\sigma+1}} d r \\
=\Psi(\sigma) \int_{0}^{\infty} \frac{N(r)}{r^{\sigma+1}} d r .
\end{aligned}
$$

From (4.12) and Lemma 2.2 we obtain

$$
\limsup _{r \rightarrow \infty} \frac{1}{N(r)} \frac{1}{2 \pi} \int_{E_{\lambda}} w\left(r e^{i \theta}\right) g(\theta) d \theta \geqslant \Psi(\lambda) .
$$

Since

$$
\begin{aligned}
\frac{1}{2 \pi} \int_{E_{\lambda}} w\left(r e^{i \theta}\right) g(\theta) d \theta & \leqslant \frac{1}{2 \pi} \int_{E_{\lambda}} w^{+}\left(r e^{i \theta}\right) g(\theta) d \theta \\
& \leqslant\left\{\frac{1}{2 \pi} \int_{-\pi}^{\pi}\left(w^{+}\left(r e^{i \theta}\right)\right)^{s} d \theta\right\}^{1 / s}
\end{aligned}
$$

we conclude from (4.13) that

$$
\limsup _{r \rightarrow \infty} \frac{m_{s}\left(r, w^{+}\right)}{N(r)} \geqslant \Psi(\lambda)=\Psi(\lambda ; g)
$$

valid for any continuous nonnegative function $g(\theta)$ that satisfies (4.7). Recalling the definition of $E_{\lambda}$ we deduce

$$
\limsup _{r \rightarrow \infty} \frac{m_{s}\left(r, w^{+}\right)}{N(r)} \geqslant m_{s}\left(\psi_{\lambda}^{+}\right)
$$

and the proof of Theorem 2 is completed.

5. Proof of Theorem 3. Let $w \in \mathscr{D}$ be of nonintegral order $\lambda$ and let $N(r)=N(r, u)+N(r, v)$. Let $r_{n}$ be the sequence of Lemma 2.1 relative to $N(r)$; then there exist numbers $\xi_{m}$ such that for a subsequence of $r_{n}$ (also denoted by $r_{n}$ ) 


$$
\frac{c_{m}\left(r_{n}\right)}{N\left(r_{n}\right)} \rightarrow \xi_{m} \quad\left(\text { all } m, r_{n} \rightarrow \infty\right)
$$

and $\xi_{m}=O\left(m^{-1}\right)$ and $\left|\xi_{m}\right| \leqslant \lambda^{2} /\left|\lambda^{2}-m^{2}\right|$.

Now if we let $\varphi(\theta) \sim \sum_{m=-\infty}^{\infty} \xi_{m} e^{i m \theta}$ and $\tilde{\varphi}(\theta)=\varphi(\theta)-\sum_{|m| \leqslant q} \xi_{m} e^{i m \theta}$ then it follows that, as in the previous theorem,

$$
\lim _{r_{n} \rightarrow \infty}\left\{\frac{1}{2 \pi} \int_{-\pi}^{\pi}\left|\frac{\tilde{w}\left(r_{n} e^{i \theta}\right)}{N\left(r_{n}\right)}-\tilde{\varphi}(\theta)\right|^{s} d \theta\right\}^{1 / s}=0 \quad(1 \leqslant s<\infty),
$$

which in turn implies that

$$
\lim _{r_{n} \rightarrow \infty} \frac{1}{N\left(r_{n}\right)} m_{s}\left(r_{n}, \tilde{w}\right)=m_{s}(\tilde{\varphi})
$$

If $s$ is a positive even integer, then a result of Hardy and Littlewood [7] together with $\left|\xi_{m}\right| \leqslant \lambda^{2} /\left(m^{2}-\lambda^{2}\right)(m \geqslant q+1)$ gives

$$
m_{s}(\tilde{\varphi}) \leqslant m_{s}\left(\sum_{|m|>q+1} \frac{\lambda^{2}}{m^{2}-\lambda^{2}} e^{i m \theta}\right)=m_{s}(\tilde{\psi} \lambda) .
$$

This completes the proof of (1.9).

REMARKS. (1) Let $u$ be subharmonic of nonintegral order $\lambda$ with its Riesz mass distributed regularly along the negative $x$-axis, i.e., $N(r) \sim r^{\lambda} L(r)$ for some slowly varying function $L$. It can be shown [1] then, that the Fourier coefficients $\left\{c_{m}(r)\right\}$ of $u$ satisfy

$$
c_{m}(r) \sim \frac{(-1)^{m} \lambda^{2}}{\lambda^{2}-m^{2}} r^{\lambda} L(r)
$$

which implies that

$$
\lim _{r \rightarrow \infty}\left\{\frac{1}{2 \pi} \int_{-\pi}^{\pi}\left|\frac{u\left(r e^{i \theta}\right)}{N(r)}-\psi_{\lambda}(\theta)\right|^{s} d \theta\right\}^{1 / s}=0 \quad(1 \leqslant s<\infty)
$$

and also

$$
\lim _{r \rightarrow \infty}\left\{\frac{1}{2 \pi} \int_{-\pi}^{\pi}\left|\frac{\tilde{u}\left(r e^{i \theta}\right)}{N(r)}-\tilde{\psi}_{\lambda}(\theta)\right|^{s} d \theta\right\}^{1 / s}=0 \quad(1 \leqslant s<\infty) .
$$

This establishes the sharpness of Theorems 1, 2 and 3.

(2) We wish to note the work of Hayman [15] for the case $s=1$ and dimensions $m \geqslant 2$, as well as Gariepy and Lewis [16] for space analogues of some theorems on meromorphic functions. 


\section{REFERENCES}

1. Faruk F. Abi-Khuzam, An abelian theorem for a class of subharmonic functions, Proc. Amer. Math. Soc. 27 (1977), 253-259.

2. Albert Baernstein, Integral means, univalent functions and circular symmetrization, Acta Math. 133 (1974), 139-169.

3. Albert Edrei, The deficiencies of meromorphic functions of finite lower order, Duke Math. J. 31 (1964), 1-22.

4. Albert Edrei and W. H. J. Fuchs, On the growth of meromorphic functions with several deficient values, Trans. Amer. Math. Soc. 93 (1959), 292-328.

5. (1960), 233-250.

6. A. M. Gorky, Concerning certain asymptotic properties of entire functions with real negative zeros, Kharkov Math. Soc. (4) 28 (1961). (Russian)

7. G. H. Hardy and J. E. Littlewood, Notes on the theory of series. (XIX): A problem concerning majorants of Fourier series, Quart. J. Math. Oxford 6 (1935), 304-315.

8. Simon Hellerstein and Jack Williamson, Entire functions with negative zeros and a problem of $R$. Nevanlinna, J. Analyse Math. 22 (1969), 233-267.

9. B. Ja. Levin, Distribution of zeros of entire functions, Transl. Math. Monographs, Vol. 5, Amer. Math. Soc., Providence, R. I., 1964.

10. Joseph Miles and Daniel F. Shea, An extremal problem in value-distribution theory, Quart. J. Math. Oxford (2) 24 (1973), 377-383.

11. R. Nevạnlinna, Le théorème de Picard-Borel et la théorie des fonctions meromorphes, Gauthier-Villars, Paris, 1930.

12. I. I. Privaloff, Sur la généralisation d'une formule de Jensen, Bull. Acad. Sciences URSS (1935), 837-847. (Russian)

13. I. P. Proskurnja, On the growth of meromorphic functions of infinite lower order, Dokl. Akad. Nauk. SSSR 209 (1973). $1-12$.

14. G. Valiron, Sur un théorème de M. Wiman, Opuscula math. A. Wiman dedicata (1930),

15. W. Hayman, Subharmonic functions in $R^{m}$, Actes Congres Internat. Math. 1970, vol. 2 , Gauthier-Villars, Paris, 1971, pp. 601-605.

16. D. Gariepy and R. Lewis, Space analogues of some theorems for subharmonic and meromorphic functions, Ark. Mat. 13 (1975), 91-105.

Department of Mathematics, Cornell University, Ithaca, New YorK 14853 